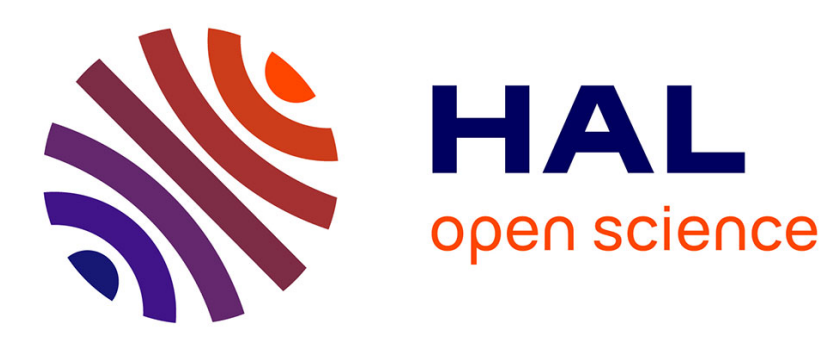

\title{
Fore-aft asymmetric flocking
}

Chen Qiu-Shi, Aurelio Patelli, Hugues Chaté, Yu-Qiang Ma, Xia-Qing Shi

\section{To cite this version:}

Chen Qiu-Shi, Aurelio Patelli, Hugues Chaté, Yu-Qiang Ma, Xia-Qing Shi. Fore-aft asymmetric flocking. Physical Review E , 2017, 96, pp.20601. 10.1103/PhysRevE.96.020601 . cea-01591666

\section{HAL Id: cea-01591666 https://hal-cea.archives-ouvertes.fr/cea-01591666}

Submitted on 21 Sep 2017

HAL is a multi-disciplinary open access archive for the deposit and dissemination of scientific research documents, whether they are published or not. The documents may come from teaching and research institutions in France or abroad, or from public or private research centers.
L'archive ouverte pluridisciplinaire HAL, est destinée au dépôt et à la diffusion de documents scientifiques de niveau recherche, publiés ou non, émanant des établissements d'enseignement et de recherche français ou étrangers, des laboratoires publics ou privés. 


\title{
Fore-aft asymmetric flocking
}

\author{
Qiu-shi Chen, ${ }^{1}$ Aurelio Patelli, ${ }^{2,3}$ Hugues Chaté, ${ }^{3,4,5}$ Yu-qiang Ma, ${ }^{1,5}$ and Xia-qing Shi ${ }^{5}$ \\ ${ }^{1}$ National Laboratory of Solid State Microstructures and Department of Physics, Nanjing University, Nanjing 210093, China \\ ${ }^{2}$ ISC-CNR, UoS Sapienza, Piazzale Aldo Moro 5, 00185 Roma, Italy \\ ${ }^{3}$ Service de Physique de l'Etat Condensé, CEA, CNRS, Université Paris-Saclay, CEA-Saclay, 91191 Gif-sur-Yvette, France \\ ${ }^{4}$ Beijing Computational Science Research Center, Beijing 100094, China \\ ${ }^{5}$ Center for Soft Condensed Matter Physics and Interdisciplinary Research, Soochow University, Suzhou 215006, China
}

(Received 14 November 2016; revised manuscript received 9 May 2017; published 14 August 2017)

\begin{abstract}
We show that fore-aft asymmetry, a generic feature of living organisms and some active matter systems, can have a strong influence on the collective properties of even the simplest flocking models. Specifically, an arbitrarily weak asymmetry favoring front neighbors changes qualitatively the phase diagram of the Vicsek model. A region where many sharp traveling band solutions coexist is present at low noise strength, below the Toner-Tu liquid, at odds with the phase-separation scenario well describing the usual isotropic model. Inside this region, a "banded-liquid" phase with algebraic density distribution coexists with band solutions. Linear stability analysis at the hydrodynamic level suggests that these results are generic and not specific to the Vicsek model.
\end{abstract}

DOI: 10.1103/PhysRevE.96.020601

Nonreciprocal (effective) interactions are interesting but rather rare in physical systems [1]. They are, however, likely to be more common in active matter. A nice example of action-reaction symmetry breaking was given recently by Soto and Golestanian for catalytically active colloids [2]. A strong case is that of self-propelled objects interacting solely via volume exclusion: their shape governs their effective interaction (e.g., aligning or not) and thus their collective behavior [3]. In the context of animal and human collective motion, asymmetric interactions are quite generic, and this asymmetry lies mostly in the relative position and weight of neighbors: the importance and quality of the information perceived by living organisms usually varies with its origin. In animal groups one often-but not always, cf. the cannibalistic behavior of locusts in Refs. [4] and [5] - expects that frontal stimuli such as neighbor positions matter more to an individual than events taking place in its back. Somewhat surprisingly, this generic fore-aft asymmetry has not been much investigated per se. It is explicitly mentioned in some works [6] and implicitly present in a number of models, see, e.g., [7], and the rather complicated escape-pursuit mechanisms introduced in Refs. [8] and [9] to describe marching locusts, or the "motion guided attention" described in Ref. [10]. It can even be found in variants of simple flocking models such as the Vicsek model, where local alignment of constant-speed particles competes with noise [11-13]. In [14-18], the introduction of a limited angle of vision was shown to have an influence on the shape of cohesive moving groups, on the degree of ordering, etc. Asymmetric interactions are also present in "metric-free" models introduced in the context of bird flocks [19-22].

In all cases mentioned above, it was not shown that fore-aft asymmetry alone can lead to qualitatively new collective phenomena. Recently, though, the influence of fore-aft asymmetric neighbors was investigated in the context of flocking models incorporating fast "inertial spin" variables [23,24]. Both these works argue that the combination of asymmetric neighbors and fast variables induces new collective behavior.

In this Rapid Communication, we show that fore-aft asymmetry alone has a strong influence on the collective properties of even the simplest flocking models, devoid of fast inertial variables. Specifically, an arbitrarily weak asymmetry favoring front neighbors changes qualitatively the phase diagram of the Vicsek model, chosen here once more for its archetypical value. The phase diagram of the usual symmetric Vicsek model—recalled in Fig. 1(e)—is now well understood [25] as the result of a phase separation between a disordered gas and a polarly ordered liquid endowed with the nontrivial correlations and fluctuations akin to those predicted by Toner and $\mathrm{Tu}$ [26]. Here we find that the microphase coexistence region is very different even for weak fore-aft asymmetry, and that the high-order high-density traveling bands characterizing it are much more robust and dominant, forcing one to reconsider the genericity of the liquid-gas phase-separation scenario. We also show the emergence, for strong-enough asymmetry, of an ordered phase different from the Toner-Tu liquid, characterized by the heavy tail of its distribution of local density. Finally, we derive hydrodynamic equations which we show to have a linear stability phase diagram in qualitative agreement with our findings at the microscopic level, which suggests that our results are generic and not specific to the Vicsek model.

Fore-aft asymmetry can be implemented in different ways. We do so via a minimal modification of the two-dimensional Vicsek model where the only new feature is that front and back neighbors have respective weights $\frac{1+\alpha}{2}$ and $\frac{1-\alpha}{2}$, so that when $\alpha=1$ (resp. -1 ) only front (resp. back) neighbors are taken into account (with equal weights). We thus consider $N$ point particles moving at constant speed $v_{0}$ in a periodic square domain of linear size $L$. At each discrete time step $\Delta t=1$, the headings $\theta$ and positions $\mathbf{r}$ of all particles are updated in parallel according to

$$
\begin{gathered}
\theta_{j}^{t+1}=\arg \left[\left\langle\omega_{j k}^{t} \exp i \theta_{k}^{t}\right\rangle_{k \in \mathcal{N}_{j}}\right]+\eta \xi_{j}^{t}, \\
\mathbf{r}_{i}^{t+1}=\mathbf{r}_{i}^{t}+v_{0} \mathbf{e}_{i}^{t+1},
\end{gathered}
$$

where $\mathcal{N}_{i}$ is the unit disk around particle $i, \xi_{i}^{t}$ a random angle drawn uniformly in $[-\pi, \pi], \eta$ sets the noise intensity, $\mathbf{e}_{i}^{t+1}$ is 
(a) $\alpha=1$

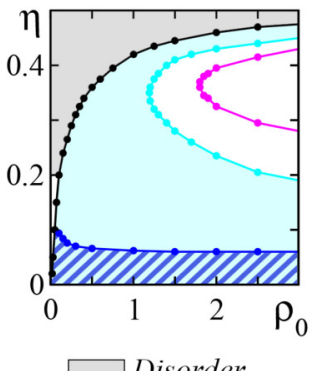

(b) $\alpha=0.6$

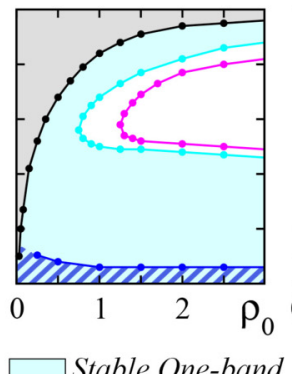

(c) $\alpha=0.3$

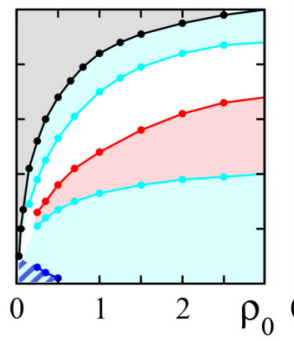

(d) $\alpha=0.1$

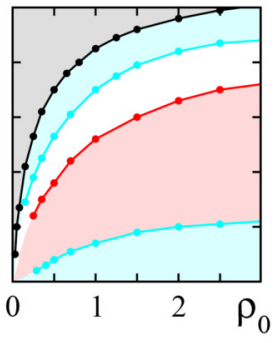

(e) $\alpha=0$ asymptotic

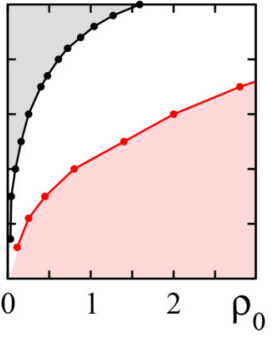

(f) $\alpha=0.3$ asymptotic

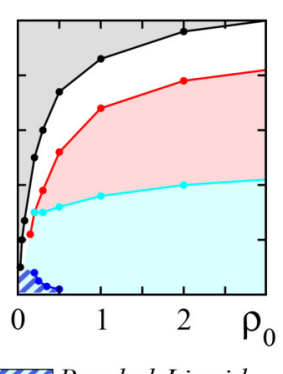

FIG. 1. Phase diagram of fore-aft asymmetric Vicsek model with front preference $(\alpha>0)$ in $\left(\rho_{0}, \eta\right)$ plane. Cyan region: stability of the one-band solution. Red region: Toner-Tu liquid phase. Blue-stripes region: banded-liquid phase. Black line: order-disorder transition. In the white regions, one observes solutions with more than one band. Blue line: upper limit of "banded-liquid" phase. Cyan line: limit of stability of the one-band solution. Magenta line: limit of stability of the two-band solution [only determined in (a), (b)]. Red line: upper limit of the Toner-Tu liquid phase. (a)-(d) finite-size systems $(L=256)$ with $\alpha=1,0.6,0.3$, and 0.1 . (e), (f) asymptotic $(L \rightarrow \infty)$ diagrams for $\alpha=0$ (usual Vicsek model, data from [25]) and $\alpha=0.3$.

the unit vector pointing in direction given by $\theta_{i}^{t+1}$, and the weights $\omega_{j k}^{t}=\frac{1}{2}\left(1+\alpha \operatorname{sign}\left[\left(\mathbf{r}_{k}^{t}-\mathbf{r}_{j}^{t}\right) \cdot \mathbf{e}_{j}^{t}\right]\right)$.

We first present results obtained in a numerical study of the model at $\alpha=1$ (only front neighbors are taken into account), varying the global density $\rho_{0}=N / L^{2}$ and the noise intensity $\eta$. The phase diagram at fixed size $L=256$ is presented in Fig. 1(a). (See numerical protocol in Appendix A.) The order-disorder transition line (black line) is similar to but slightly lower than that of the symmetric model, whose phase diagram is shown in Fig. 1(e). In strong contrast to this last case, there is no region of homogeneous Toner-Tu liquid at low noise strength. Instead, traveling band solutions, characteristic of the coexistence phase, are observed everywhere below the order-disorder line. More specifically, the solution containing a single band is stable in the cyan region between the orderdisorder line and the C-shaped cyan line in Fig. 1(a). Similarly the two-band solution is stable from almost the order-disorder line until the $\mathrm{C}$-shaped magenta line [27]. In the low noise blue-stripes region below the blue line, a phase that we call "banded liquid" (see below and Fig. 2) coexists with many stable band solutions. Exploring very large values of $\rho_{0}$, we find that the lower branch of the $\mathrm{C}$-shaped curves and the line delimiting the banded liquid become largely independent of $\rho_{0}$. Above the C-shaped curves, at densities $\rho_{0} \gtrsim 20$, we observed what could be the usual Toner-Tu liquid (not shown), even though it is hard to reach solid conclusions for such parameters.

One striking feature in the above observations is the robustness of the band solutions, and their extremely sharp fronts and small width in the low noise region. For $\eta=0.1$, for instance, the one-band solution seems stable for arbitrarily large $\rho_{0}$, reaching ever-higher peak densities as $\rho_{0}$ increases [Figs. 3(a) and 3(b)]. This solution is also stable for arbitrary large system size $L$, all parameters being fixed [Figs. 3(c) and 3(d)]. These observations invalidate in practice the liquid-gas scenario (which implies that the number of bands scales linearly with $\rho_{0}$ and/or $L$ [25]). Nevertheless, we are not in the presence of some "condensation" phenomenon: two bands at short distance from each other do experience mutual repulsion, leading them to be asymptotically equidistant (not shown).

We now turn to a description of the "banded liquid." Like the ordered liquid of the symmetric model, this phase shows true long-range polar order (not shown) and exhibits "giant" anomalous number fluctuations with approximately the same scaling exponent [Fig. 2(c)]. But unlike the Toner-Tu liquid, it always coexists with band solutions [28]. Moreover, as seen from Fig. 2(a), extremely high density packets are present, and the bandlike structures to which they belong are only seen if (a)

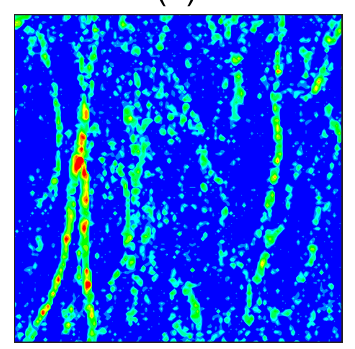

(c)

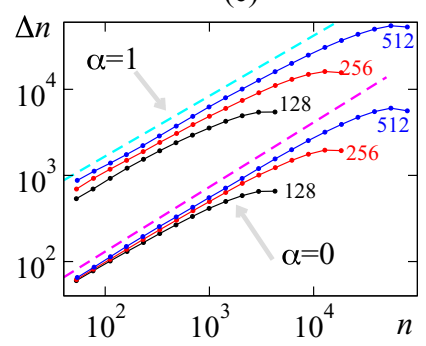

(b)

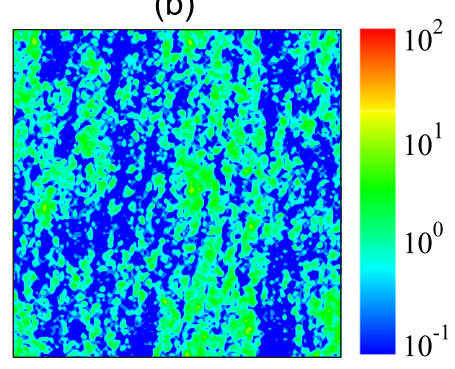

(d)

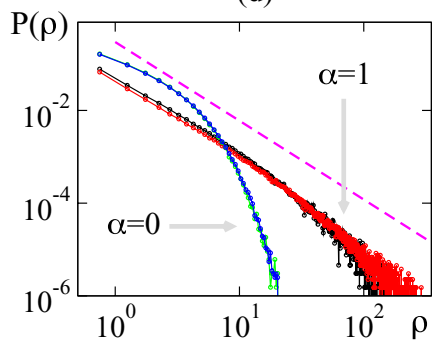

FIG. 2. Banded-liquid phase observed for $\alpha=1, \rho_{0}=1, \eta=$ 0.055. (a), (b) Snapshots of density field calculated over square boxes of linear size $\ell=4$ in a system of size $L=512$ (logarithmic color scale: (a) pure front model $\alpha=1$ and (b) Toner-Tu liquid of symmetric Vicsek model $\alpha=0$. (c) Giant number fluctuations: rms $\Delta n$ of number of particles $n$ contained in boxes of various linear sizes. Top three curves (shifted up for clarity): pure front model $(\alpha=1)$. Bottom three curves: symmetric Vicsek model $(\alpha=0)$. Dashed cyan (resp. magenta) line is a power law of exponent 0.7 (resp. 0.75). The system size is indicated near each curve. (d) Probability distribution function of coarse-grained density (black and red lines: $\alpha=1, L=256,512$; green and blue lines: $\alpha=0, L=256,512$ ). Dashed magenta line is a power law of slope -1.7 . 
(a)

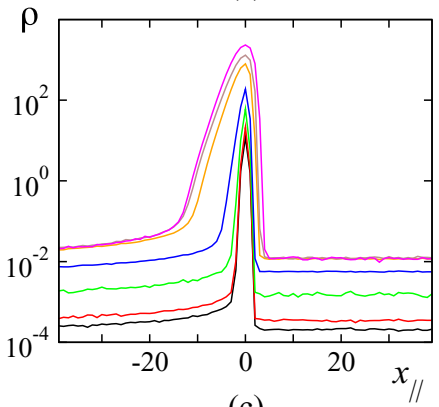

(c)

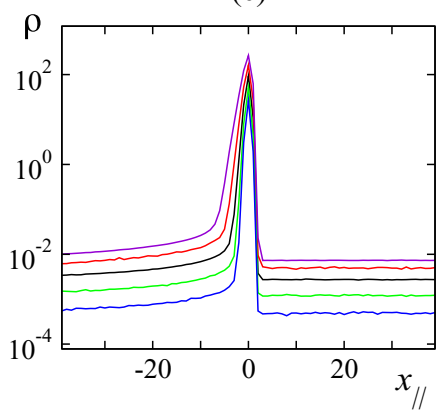

(b)

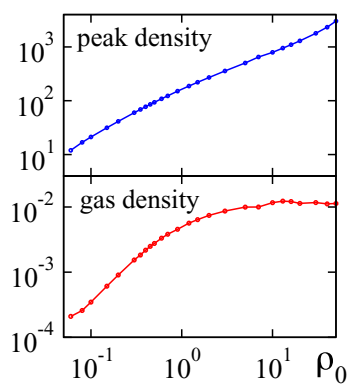

(d)

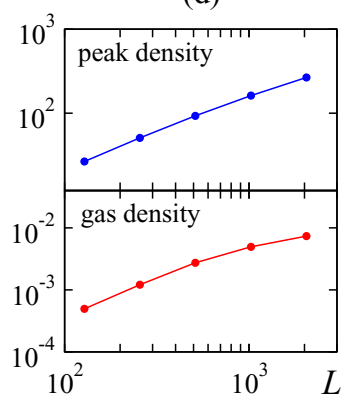

FIG. 3. Robustness of the one-band solution $(\alpha=1, \eta=0.1)$. (a) Time-averaged density profiles at various $\rho_{0}$ values. From bottom to top: $\rho_{0}=0.06,0.1,0.3,1.2,10,20,50(L=256)$. (b) Peak density (top) and gas density (bottom) vs $\rho_{0}(L=256)$. (c) Time-averaged profiles for $L=128,256,512,1024,2048$ from bottom upward $\left(\rho_{0}=\right.$ 0.25). (d) Same as (b) but for profiles of (c).

some logarithmic density scale is adopted. This is in contrast with the usual Toner-Tu liquid phase, a snapshot of which is shown in Fig. 2(b) for comparison. Looking at the distribution function of (coarse-grained) density, a qualitative difference between the two ordered liquids appears: the banded liquid exhibits a power-law tail with exponent $\sim 1.7$, whereas only an approximately exponential tail is seen for the Toner-Tu liquid [Fig. 2(d)].

The results presented so far show that restricting neighbors to those strictly in front $(\alpha=1)$ induces strong differences with the usual $(\alpha=0)$ Vicsek model. This is not due to some singularity of the $\alpha=1$ case: decreasing $\alpha$ from 1 to 0 , the phase diagram changes smoothly [Figs. 1(a)-1(d)]: the banded-liquid region (blue stripes) shrinks to some triangle near the origin of the $\left(\rho_{0}, \eta\right)$ plane and vanishes completely for $\alpha \lesssim 0.25$. The $\mathrm{C}$-shaped curves delimiting the stability of the one-band and two-band solutions move towards the origin and open up into two separate regions for $\alpha \lesssim 0.4$, while the Toner-Tu liquid appears in a central red region. Approaching $\alpha=0$, the lower region of stability of the one-band solution recedes to larger and larger densities, leaving the familiar phase diagram of the symmetric Vicsek model [Fig. 1(e)].

We now present evidence that our main findings hold in the infinite-size limit. For that numerically demanding task, we focused on the $\alpha=0.3$ case (see Appendix A for details). Crossing the blue lines from below in Fig. 1, the banded liquid disappears in a clearly discontinuous transition, typically leaving many thin bands. We located this transition at fixed $\rho_{0}$ for systems of increasing size. The transition values $\eta_{\mathrm{bl}}(L)$ thus defined increase with $L$ and converge to a finite asymptotic value exponentially, $\eta_{\mathrm{bl}}(\infty)-\eta_{\mathrm{bl}}(L) \approx \exp (-k L)$, as expected in first-order phase transitions. Repeating this for different $\rho_{0}$ values, we find that the banded-liquid region converges to the blue line in Fig. 1(f). (For larger $\alpha$ values, the asymptotic banded-liquid region remains unbounded in $\rho_{0}$.)

Similarly, we tracked the stability region of the one-band solution (again, some details are found in Appendix A). We found that its lower part converges to a well-defined domain delimited by the cyan line in Fig. 1(f). The upper part of the stability region of the one-band solution behaves like in the $(\alpha=0)$ Vicsek model: it quickly converges onto the orderdisorder line as $L$ increases [and is thus undistinguishable from the black order-disorder line in Fig. 1(f)]. As for the symmetric model, there exists, nevertheless, a coexistence region containing smectic arrangements of band solutions [white area in Fig. 1(f)]. We thus conclude that the asymptotic phase diagram of our model for $\alpha=0.3$ is qualitatively different from that of the symmetric model [compare Figs. 1(e) and 1(f)]. It comprises an extra banded-liquid phase as well as a second region supporting band solutions that seem unbounded to the right.

In fact, the phase diagram seems to comprise a lower band region for any finite $\alpha$ value: the one-band solution can be found stable for values as low as $\alpha=0.01$, provided that $\rho_{0}$ is large enough. For instance, working at fixed low noise $\eta=0.03$ and $L=256$, we find that the one-band solution is stable for at least millions of time steps for $\rho_{0} \geqslant \rho^{*}(\alpha)$, with $\rho^{*} \simeq 0.65,1.1,1.8,3$ for $\alpha=0.06,0.04,0.02,0.01$ and that $\rho_{0}^{*}$ decreases with increasing system size (at fixed $\alpha$ ). This suggests nothing less than the singularity and "fragility" of the classic Vicsek model_ and thus, presumably, of many flocking models in its class - with respect to fore-aft asymmetry.

We finally report evidence of the robustness of our findings. First, they are not specific to the variant of the Vicsek model defined by Eqs. (1) and (2): implementing instead a restricted angle of vision $\left[\theta_{i}-\delta ; \theta_{i}+\delta\right]$ around the particle's headings $\theta_{i}$, we found phase diagrams similar to those of Fig. 1 for $\delta \in$ $\left[\frac{\pi}{2}, \pi\right]$ (not shown). Next, we now show briefly how one can account for our results at the hydrodynamic level (details can be found in Appendix B). We use a variant of the BoltzmannGinzburg-Landau method used successfully for Vicsek-style models [29]. This approach, or for that matter any one based on a controlled truncation and closure of a kinetic equation governing the one-body distribution function $f(\theta, \vec{r}, t)$, can only produce the following Toner-Tu equations coupling the density and the ordering fields $\rho$ and $\mathbf{w}=\rho \mathbf{P}$ (where $\mathbf{P}$ is the polar order field):

$$
\begin{gathered}
\partial_{t} \rho+\nabla \cdot \mathbf{w}=D \Delta \mathbf{w} \\
\partial_{t} \mathbf{w}+\frac{1}{2} \nabla \rho=\left[\mu-\xi|\mathbf{w}|^{2}\right] \mathbf{w}+D_{\mathrm{i}} \Delta \mathbf{w}+D_{\mathrm{a}} \nabla(\nabla \cdot \mathbf{w}) \\
-\lambda_{1}(\mathbf{w} \cdot \nabla) \mathbf{w}-\lambda_{2}(\nabla \cdot \mathbf{w}) \mathbf{w}-\lambda_{3} \nabla|\mathbf{w}|^{2}
\end{gathered}
$$

but the explicit form of the $\rho$-dependent transport coefficients does depend on the precise kinetic equation used. Here we write a standard Boltzmann equation with effective positional 
(a)

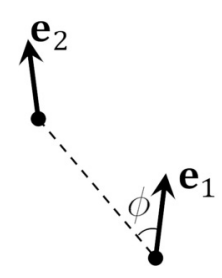

(b)

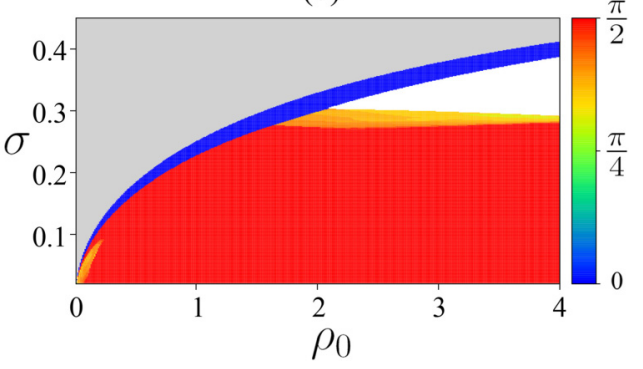

FIG. 4. (a) Sketch of the geometry of interaction between particles where particle 1 is taken as the reference particle. (b) Phase diagram in the $\left(\rho_{0}, \sigma\right)$ plane showing the linear stability analysis of the homogeneous ordered solution $|\mathbf{w}|^{2}=\mu / \xi$ of Eqs. (3) and (4). The color coding indicates the direction of the most unstable mode compared to that of the polar order. In the white region, the homogeneous order is stable; the gray region denotes the stable disordered phase.

diffusion [30] and the collision integral:

$$
\begin{aligned}
I_{\mathrm{col}}[f]= & \int d \theta_{1} d \theta_{2} f\left(\theta_{1}, \vec{r}\right) f\left(\theta_{2}, \vec{r}\right) \int d \phi K\left(\theta_{2}-\theta_{1}, \phi\right) \\
& \times\left\{P_{\eta} * \delta\left[\Psi\left(\theta_{1}, \theta_{2}\right)\right]-\delta\left(\theta_{1}-\theta\right)\right\}
\end{aligned}
$$

where $P_{\eta}$ is the angular noise distribution, $\Psi\left(\theta_{1}, \theta_{2}\right)=$ $\arg \left\{\exp \left[\frac{i}{2}\left(\theta_{1}+\theta_{2}\right)\right]\right\}$ the polar alignment function, and $*$ the convolution operator. The main difference with respect to the traditional modeling of collisions is encoded in the kernel $K\left(\theta_{2}-\theta_{1}, \phi\right)$ and the integral over $\phi$, the angular position of particle 2 in the reference frame of particle 1 [see Fig. 4(a)]. Following the usual route, collisions would be restricted to those with particles ahead approaching the reference particle and would hence discard the crucial, persistent, aligning events with front particles going "away" from the focus particle. Here, on the contrary, the structure of $K$ favors those collisions, something we encode in the following compact expression:

$$
K(\Delta, \phi)=|\sin (\Delta / 2)|[\Phi(\Delta, \pi, \phi)+\Phi(\pi, \Delta, \phi+\pi)],
$$

where $\Phi(a, b, x)=\Theta(a-b) \Theta\left(x-\frac{a}{2}\right) \Theta\left(\frac{b}{2}-x\right)$ with $\Theta$ the Heaviside step function [31].

The resulting expressions for the transport coefficients of Eqs. (3) and (4) are given by Eqs. (B2)-(B8) in Appendix B. The study of the existence and linear stability of the homogeneous solutions of Eqs. (3) and (4) is summarized in the $\left(\rho_{0}, \sigma\right)$ phase diagram presented in Fig. 4(b), where $\sigma$ is the rms of the microscopic noise $P_{\eta}$. Similar to the microscopic model, the basic order-disorder transition line defined by $\mu=0$ is lower than the one found with symmetric interactions. Bordering this line, we find the usual longitudinal, "banding" instability of the ordered solution $|\mathbf{w}|^{2}=\mu / \xi$, but also, at low noise, a new, large, transversal instability region which almost suppresses the longitudinal instability at low densities. The exchange of instability direction is in fact the result of growing "transverse" lobes in Fourier space (see Appendix B). The new transverse instability causes the Toner-Tu liquid to be unstable at low densities, in agreement with the phase diagram of the microscopic model. Investigating whether this agreement carries over to the nonlinear and fluctuating level and possibly reveals the existence of a banded-liquid regime for the stochastic version of hydrodynamic equations (3) and (4) is a difficult task left for future studies. Preliminary simulations at the deterministic level are encouraging in this respect: in the transverse instability in Fig. 4(b), we observe very sharp band solutions, reminiscent of those observed in the microscopic model.

To summarize, even weak fore-aft asymmetry, a generic feature of living organisms and some active matter systems, can have a strong qualitative influence on the collective properties of even the simplest flocking models. Specifically, an arbitrarily small asymmetry favoring front neighbors changes qualitatively the phase diagram of the Vicsek model. A region where many sharp traveling band solutions coexist is present at low noise strength, below the usual Toner-Tu liquid, an observation that forces one to revisit the phase-separation scenario put forward for symmetric flocking models. Inside this region, a banded-liquid phase with algebraic density distribution coexists with the band solutions. Stability analysis at the hydrodynamic level suggests that these results are generic and not specific to the Vicsek model.

Future work will try to connect our results to the interesting predictions made in Refs. [23] and [24] for asymmetric models with fast underdamped inertial spin variables. More generally, our results indicate that nonreciprocal interactions in active matter deserve further study.

\section{ACKNOWLEDGMENT}

This work is supported by the National Natural Science Foundation of China (No. 11635002 to H.C. and X.S.; No. 91427302 and No. 11474155 to Y.M.; No. 11474210 and No. 11674236 to X.S.).

\section{APPENDIX A: NUMERICAL PROTOCOLS AND DETAILS}

Numerical protocol for the phase diagrams in Figs. 1(a)$1(d)$. In the cyan region, the one-band solution is observed
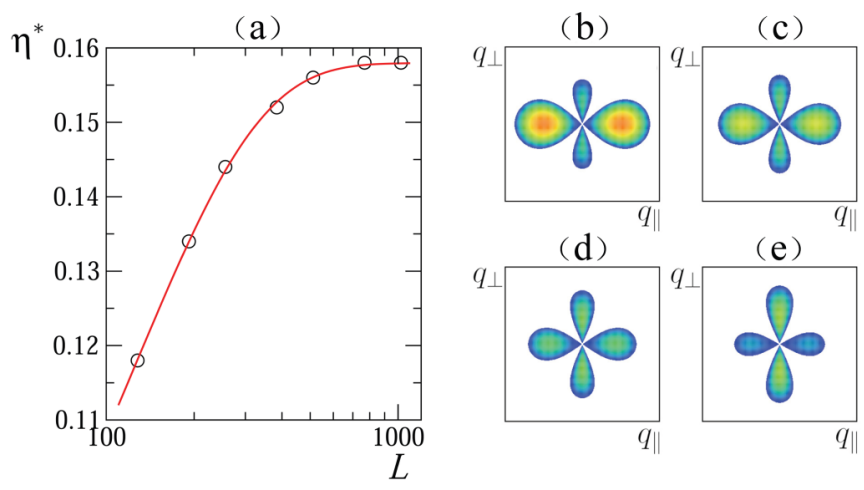

(e)

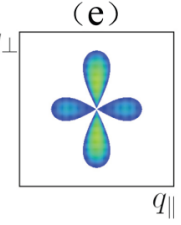

FIG. 5. (a) Convergence with system size of the transition point $\eta^{*}(L)$ delimiting the lower part of the stability region of the oneband solution $\left(\alpha=0.3, \rho_{0}=0.5\right)$. The red solid line is a fit $\eta^{*}(L)=$ $\eta_{\infty}^{*}-a \exp (-b L)$ with $\eta_{\infty}^{*} \simeq 0.158$. (b)-(e) Unstable modes for the homogeneous solution $|\mathbf{w}|^{2}=\mu / \xi$ at $\rho_{0}=1.5$ in the $\left(q_{\|}, q_{\perp}\right)$ plane $\left(q_{\|}, q_{\perp} \in[-0.025,0.025]\right)$. Color codes for the growth rate only if it is positive, from blue $=0$ to red $=10^{-3}$ : (b) $\sigma=0.267$, (c) $\sigma=0.265$, (d) $\sigma=0.263$, and (e) $\sigma=0.261$. 
for at least $10^{6}$ time steps. Similarly, the two-band solution is observed for at least $10^{6}$ time steps left of the magenta line in Figs. 1(a) and 1(b). The black, cyan, and magenta transition lines obtained are sharply defined because the lifetime of the one-band and two-band solutions quickly and surely becomes very short across them. The blue line marking the limit of the banded-liquid phase is again defined by the condition that the banded liquid is observed for at least $10^{6}$ time steps. The corresponding transition is sharp, as the breakdown of the banded liquid quickly leads to a solution containing a number of very thin bands, but it has all the features of a first-order phase transition. In particular, the breakdown of the banded liquid follows the nucleation of some local thin band(s). Our protocol to locate the transition points was that, over 100 runs, at least 50 of them led to band solutions within $10^{6}$ time steps.

Convergence of transition points at fixed parameters as $L \rightarrow \infty$ [Fig. 1(f)]. to determine each of the transition points shown in the asymptotic phase diagram in Fig. 1(f), we followed the same protocol as outlined above for different system sizes. This yielded a series of transition values which typically converges exponentially to a finite asymptotic value. An example is shown in Fig. 5(a).

\section{APPENDIX B: DERIVATION OF HYDRODYNAMIC EQUATIONS AND LINEAR STABILITY ANALYSIS}

Derivation of hydrodynamic equations. The Boltzmann kinetic equation describing the evolution of the single particle distribution function $f=f(\mathbf{r}, \theta, t)$ reads

$$
\partial_{t} f+v_{0} \mathbf{e}(\theta) \cdot \vec{\nabla} f=D \Delta f+I_{\mathrm{dif}}[f]+I_{\mathrm{col}}[f],
$$

where $\mathbf{e}(\theta)$ is the unit vector along $\theta$ and $I_{\mathrm{dif}}[f]=-f+\left(P_{\eta} *\right.$ $f$ ) is the angular self-diffusion integral, with $P$ the distribution of the angular noise and $*$ the convolution operator. In addition to this rotational diffusion, we consider also spatial diffusion expressed by the term $D \triangle f$. Our Vicsek-style model does not have explicit positional diffusion, but its discrete-time dynamics generates it. Here, we introduce it explicitly mostly because this term, with $D$ large enough, removes the spurious instability of the homogeneous state found away from the orderdisorder line in the resulting hydrodynamic equations [29].

For the standard (isotropic) Vicsek model, the collision between two particles can happen along any approaching direction. For the "front-biased" model $(\alpha>0)$, in contrast, we favor the "dominant" aligning interactions, which are those taking place effectively with particles already following the reference particle. These collisions are frequent, the relative Velocity of the two particles is small, when the relative direction of particle 2 lies in $\phi \in[\pi / 2,3 \pi / 2]$. This is encoded in the $K\left(\theta_{2}-\theta_{1}, \phi\right)$ given in the main text.

The hydrodynamic equations are then obtained following the usual procedure: expanding the Boltzmann equation in Fourier series of the angular variable $\theta,[f(\mathbf{r}, \theta, t)=$ $\left.\sum_{-\infty}^{\infty} f_{k}(\mathbf{r}, t) \exp (i k \theta)\right]$, leading to a hierarchy of partial differential equations governing the fields $f_{k}$. Using a propagative scaling ansatz [29], this hierarchy is truncated and closed, leading to the usual Toner-Tu equations given in the text with the following transport coefficients:

$$
\begin{gathered}
\mu(\rho)=P_{1}-1+\frac{1}{12 \pi}\left[8 P_{1}-(16-3 \pi)\right] \rho, \\
\xi=-\frac{8 P_{1}+15 \pi-48}{120 \pi^{2} \mu_{2}}\left[\frac{4}{3}-\frac{\pi}{2}+(4-\pi) P_{2}\right], \\
D_{i}=D-\frac{1}{4 \mu_{2}} \quad D_{a}=0, \\
\lambda_{1}=-\kappa_{2}-\kappa_{1} \quad \lambda_{2}=-\kappa_{2}+\kappa_{1} \quad \lambda_{3}=-\frac{\lambda_{2}}{2},
\end{gathered}
$$

with

$$
\begin{gathered}
\mu_{2}=P_{2}-1-\frac{1}{2 \pi}\left[\left(\frac{8}{3}-\pi\right) P_{2}+\left(\frac{56}{15}-\pi\right)\right] \rho_{0}, \\
\kappa_{1}=\frac{1}{2 \pi \mu_{2}}\left[\frac{4}{3}-\frac{\pi}{2}+(4-\pi) P_{2}\right] \\
\kappa_{2}=-\frac{1}{120 \pi \mu_{2}}\left(8 P_{1}+15 \pi-48\right)
\end{gathered}
$$

where $P_{k}=\int_{-\infty}^{\infty} d \sigma P_{\eta}(\sigma) \exp (i k \sigma)$.

Linear stability analysis of the ordered solution. The homogeneous ordered solution $|\mathbf{w}|^{2}=\mu / \xi$ to the hydrodynamic equations is unstable in a large region. Near the order-disorder transition line, the most unstable modes are longitudinal. Decreasing $\sigma$, the most unstable modes become transversal ones. In Figs. 5(b)-5(e), we show how this happens in the $\left(q_{\|}, q_{\perp}\right)$ Fourier space of perturbations. Only modes with positive growth rate are shown. They form four "lobes" whose relative strength varies with $\sigma$.
[1] A. V. Ivlev, J. Bartnick, M. Heinen, C.-R. Du, V. Nosenko, and H. Löwen, Phys. Rev. X 5, 011035 (2015).

[2] R. Soto and R. Golestanian, Phys. Rev. Lett. 112, 068301 (2014); Phys. Rev. E 91, 052304 (2015).

[3] H. H. Wensink, V. Kantsler, R. E. Goldstein, and J. Dunkel, Phys. Rev. E 89, 010302(R) (2014).

[4] S. J. Simpson, G. A. Sword, P. D. Lorch, and I. D. Couzin, Proc. Natl. Acad. Sci. USA 103, 4152 (2006).

[5] S. Bazazi, J. Buhl, J. J. Hale, M. L. Anstey, G. A. Sword, S. J. Simpson, and I. D. Couzin, Curr. Biol. 18, 735 (2008).

[6] L. Barberis and F. Peruani, Phys. Rev. Lett. 117, 248001 (2016).
[7] R. Lukeman, Y.-X. Li, and L. Edelstein-Keshet, Proc. Natl. Acad. Sci. USA 107, 12576 (2010).

[8] P. Romanczuk, I. D. Couzin, and L. Schimansky-Geier, Phys. Rev. Lett. 102, 010602 (2009).

[9] P. Romanczuk and L. Schimansky-Geier, Interface Focus 2, 746 (2012).

[10] B. H. Lemasson, J. J. Anderson, and R. A. Goodwin, Proc. R. Soc. London, Ser. B 280, 20122003 (2013).

[11] T. Vicsek, A. Czirok, E. Ben-Jacob, I. Cohen, and O. Shochet, Phys. Rev. Lett. 75, 1226 (1995).

[12] G. Grégoire and H. Chaté, Phys. Rev. Lett. 92, 025702 (2004). 
[13] H. Chaté, F. Ginelli, G. Grégoire, and F. Raynaud, Phys. Rev. E 77, 046113 (2008).

[14] X.-G. Wang, C.-P. Zhu, C.-Y. Yin, D.-S. Hu, and Z.-J. Yan, Phys. A (Amsterdam, Neth.) 392, 2398 (2013).

[15] B.-M. Tian, H.-X. Yang, W. Li, W.-X. Wang, B.-H. Wang, and T. Zhou, Phys. Rev. E 79, 052102 (2009).

[16] Y.-J. Li, S. Wang, Z.-L. Han, B.-M. Tian, Z.-D. Xi, and B.-H. Wang, Europhys. Lett. 93, 68003 (2011).

[17] P. T. Nguyen, S.-H. Lee, and V. T. Ngo, Phys. Rev. E 92, 032716 (2015).

[18] M. Durve and A. Sayeed, Phys. Rev. E 93, 052115 (2016).

[19] M. Ballerini, N. Cabibbo, R. Candelier, A. Cavagna, E. Cisbani, I. Giardina, V. Lecomte, A. Orlandi, G. Parisi, A. Procaccini, M. Viale, and V. Zdravkovic, Proc. Natl. Acad. Sci. USA 105, 1232 (2008).

[20] F. Ginelli and H. Chaté, Phys. Rev. Lett. 105, 168103 (2010).

[21] M. Camperi, A. Cavagna, I. Giardina, G. Parisi, and E. Silvestri, Interface Focus 2, 715 (2012).

[22] D. J. G. Pearce, A. M. Miller, G. Rowlands, and M. S. Turner, Proc. Natl. Acad. Sci. USA 111, 10422 (2014).

[23] A. Cavagna, I. Giardina, A. Jelic, S. Melillo, L. Parisi, E. Silvestri, and M. Viale, Phys. Rev. Lett. 118, 138003 (2017).
[24] L. P. Dadhichi, R. Chajwa, A. Maitra, and S. Ramaswamy, arXiv:1605.00981.

[25] A. P. Solon, H. Chaté, and J. Tailleur, Phys. Rev. Lett. 114, 068101 (2015).

[26] J. Toner and Y. Tu, Phys. Rev. Lett. 75, 4326 (1995); Phys. Rev. E 58, 4828 (1998); J. Toner, ibid. 86, 031918 (2012).

[27] There is thus a large region of parameter space where both the one-band and the two-band solutions can be observed, depending on initial conditions.

[28] It is only reached from certain classes of initial conditions, for instance, initial configurations with random locations but ordered orientation.

[29] A. Peshkov, E. Bertin, F. Ginelli, and H. Chaté, Eur. Phys. J. Spec. Top. 223, 1315 (2014).

[30] The "spurious instability" found at low noise at hydrodynamic level in the symmetric case without positional diffusion was found to disappear upon the addition of even weak diffusion [29]. Here diffusion has the same regularizing effect, and we thus add it "by hand."

[31] Note that this choice stands for the extreme case $\alpha=1$. Interpolating between this and the usual symmetric neighbors case, $K(\Delta)=|\sin (\Delta / 2)|$, is left for future work. 